\title{
Amentoflavone Inhibits ERK-modulated Tumor Progression in Hepatocellular Carcinoma In Vitro
}

\author{
KUN-CHING LEE ${ }^{1,2}$, JAI-JEN TSAI ${ }^{3}$, CHIH-WEI TSENG $^{4}$, YU-CHENG KUO ${ }^{5,6}$, \\ YAO-CHEN CHUANG ${ }^{7}$, SONG-SHEI LIN ${ }^{1 *}$ and FEI-TING HSU $^{8-10^{*}}$ \\ ${ }^{1}$ Department of Medical Imaging and Radiological Sciences, \\ Central-Taiwan University of Science and Technology, Taichung, Taiwan, R.O.C.; \\ ${ }^{2}$ Department of Radiation Oncology, National Yang-Ming University Hospital, Yilan, Taiwan, R.O.C.; \\ ${ }^{3}$ Division of Gastroenterology, Department of Medicine, \\ National Yang-Ming University Hospital, Yilan, Taiwan, R.O.C.; \\ ${ }^{4}$ Division of Gastroenterology, Department of Internal Medicine, Dalin Tzu Chi Hospital, \\ Buddhist Tzu Chi Medical Foundation, Chia-Yi, Taiwan, R.O.C.; \\ ${ }^{5}$ Radiation Oncology, China Medical University Hospital, Taichung, Taiwan, R.O.C.; \\ ${ }^{6}$ Department of Radiation Oncology, Show Chwan Memorial Hospital, Changhua, Taiwan, R.O.C.; \\ ${ }^{7}$ Kiang Wu Nursing College of Macau, Macau SAR, P.R. China; \\ ${ }^{8}$ Department of Radiology, School of Medicine, College of Medicine, \\ Taipei Medical University, Taipei, Taiwan, R.O.C.; \\ ${ }^{9}$ Department of Medical Imaging, Taipei Medical University Hospital, \\ Taipei Medical University Hospital, Taipei, Taiwan, R.O.C.; \\ ${ }^{10}$ Research Center of Translational Imaging, College of Medicine, Taipei Medical University, Taipei, Taiwan, R.O.C.
}

\begin{abstract}
Background/Aim: A previous study indicated that amentoflavone inhibits tumor growth of breast cancer. However, the anti-cancer effects and mechanism of amentoflavone in hepatocellular carcinoma (HCC) have not been elucidated. The aim of the present study was to verify the effect of amentoflavone on tumor progression in HCC. Materials and Methods: HCC SK-Hepl cells were treated with different concentrations of amentoflavone or 10 MM PD98059 (extracellular signal-regulated kinases (ERK) inhibitor) for $48 \mathrm{~h}$, respectively, and then cell viability, $N F-\kappa B$ activation, levels of tumor progression-
\end{abstract}

This article is freely accessible online.

*These Authors contributed equally to this study.

Correspondence to: Song-Shei Lin and Fei-Ting Hsu, , Song-Shei Lin, Department of Medical Imaging and Radiological Sciences, CentralTaiwan University of Science and Technology, Taichung, Taiwan, R.O.C. Tel: +886 422391647 \#7111, e-mail: sslin@ctust.edu.tw; and Fei-Ting Hsu, Department of Medical Imaging, Taipei Medical University Hospital, No. 252 Wu Hsing Street, Taipei, Taiwan 110 R.O.C. Tel: +886 227372181\#1133, e-mail: sakiro920@tmu.edu.tw

Key Words: Amentoflavone, hepatocellular carcinoma, NF-кB, ERK. associated proteins, and cell invasion were evaluated with 3-(4,5-Dimethylthiazol-2-yl)-2,5-diphenyltetrazolium bromide (MTT), NF-кB reporter gene assay, western blotting, and cell invasion assay. Results: The results demonstrated that both amentoflavone and PD98059 not only significantly reduced cell viability, $N F-k B$ activation, and cell invasion, but also inhibited the expression of tumor progression-associated proteins. In addition, we found that amentoflavone suppresses ERK phosphorylation. Conclusion: The results of the present study suggest that amentoflavone down-regulates ERK-modulated tumor progression in HCC.

Extracellular molecules, such as growth factors and hormones, trigger mitogen-activated protein kinase (MAPK) cascade-modulated normal cell proliferation, survival, and differentiation. Aberrant MAPK cascade is linked to cancer and other diseases. MAPK extracellular signal-regulated kinase (ERK) is phosphorylated by Raf/MAPK/ERK kinase (MEK)1/2 dual-specificity protein kinase and is associated with overexpression of oncogenic proteins that result in tumor progression (1-2). In hepatocellular carcinoma (HCC) tissues, increased expression of phospho-ERK (pERK) was correlated with poor disease-specific overall survival (3). Development of new anti-HCC agents that block ERK activation may provide benefits in treatment of HCC. 
Nuclear factor-kappaB (NF-kB), a critical transcription factor, regulates the expression of many oncogenes in tumorigenesis. Active NF-kB promotes tumor growth, antiapoptosis, angiogenesis, and metastasis of HCC through overexpression of tumor progression-associated proteins encoded by NF-kB-targeted genes (4-5). The MAPK cascade has been shown to activate NF-kB-modulated tumor progression in various cancer cells. Blockage of ERK activation may downregulate NF-kB-modulated tumor progression in $\mathrm{HCC}$ (6-8).

Amentoflavone, a polyphenolic compound that exists in many plants, has been indicated to induce anti-inflammation, anti-cancer effects, and protection of cardiovascular and central nervous system (9). In a previous study, we found amentoflavone, as a sorafenib sensitizer, which enhances sorafenib-induced cytotoxicity and apoptosis in sorafenibresistant HCC (10). However, the anti-cancer effects and mechanism of amentoflavone in HCC are ambiguous. Therefore, the aim of the present study was to verify the effect of amentoflavone on tumor progression in HCC. The role of ERK inactivation on amentoflavone-induced inhibition of tumor progression was also investigated.

\section{Materials and Methods}

Chemicals and reagents. Amentoflavone, MTT, and Dimethyl sulfoxide (DMSO) were obtained from Sigma-Aldrich (St. Louis, MO, USA). Dulbecco's modified Eagle's medium (DMEM), fetal bovine serum (FBS), L-glutamine and penicillin-streptomycin (PS) were purchased from Gibco/Life Technologies (Carlsbad, CA, USA). Hygromycin was from Santa Cruz Biotechnology (Santa Cruz, CA, USA). jetPEI-DNA transfection reagent was from Polyplus Transfection (Sélestat, Bas-Rhin, France). Dluciferin was obtained from Promega (Madison, WI, USA). Extracellular signal-regulated kinase (ERK) inhibitor PD98059 was boughtfrom Selleckchem (Houston, TX, USA). Primary antibodies of cellular FADD-like IL- $1 \beta$-converting enzyme (FLICE)-inhibitory protein (C-FLIP), and Cyclin-D1 purchased from Cell Signaling Technology (Beverly, MA, USA). A primary antibody of X-linked inhibitor of apoptosis protein (XIAP) was obtained from Thermo Fisher Scientific (Fremont, CA, USA). Primary antibodies for matrix metallopeptidase (MMP 9) and vascular endothelial growth factor (VEGF) were obtained from EMD Millipore (Billerica, MA, USA). Primary antibodies of phosphorylated extracellular signal-regulated kinase (pERK) and ERK were obtained from Merck Millipore (Billerica, MA, USA) and Santa Cruz Biotechnology (Santa Cruz, CA, USA), respectively. Matrigel was obtained from Corning (Tewksbury, MA, USA).

Cell culture. HCC SK-Hep1 cells were provided by Professor JingGung Chung at the Department of Biological Science and Technology, China Medical University, Taichung and used to verify anti-HCC effect of amentoflavone. Cells were maintained in DMEM containing 10\% FBS, $2 \mathrm{mM}$ L-glutamine, $100 \mathrm{U} / \mathrm{ml}$ penicillin, and $100 \mathrm{mg} / \mathrm{ml}$ streptomycin in a humidified incubator at $37^{\circ} \mathrm{C}$ in $5 \%$ $\mathrm{CO}_{2}$ and $95 \%$ air (11).
3-(4,5-Dimethylthiazol-2-yl)-2,5-diphenyltetrazolium bromide(MTT) assay. SK-Hep1 cells were seeded into 96-well plates with $2 \times 104 /$ well and incubated overnight. Cells were treated with different concentrations of amentoflavone or PD98059 (ERK inhibitor) for 48 $\mathrm{h}$, respectively, and then cell viability was evaluated with MTT assay, as described by Chen et al. (12).

Plasmid transfection. NF-kB-luciferase reporter plasmid (pNF-kB/luc2) was obtained from Promega (Madison, WI, USA). $1 \times 10^{6}$ SK-Hep1 cells were cultured overnight in $10 \mathrm{~cm}$ dish, and then transfected with $\mathrm{pNF}-\mathrm{kB} / \mathrm{luc} 2$ by using jetPEI-DNA transfection reagent as previously described (13).

$N F-k B$ reporter gene assay. SK-Hep1 cells were seeded into 96-well plates $\left(2 \times 10^{4} /\right.$ well $)$ and incubated overnight. Cells were treated with different concentrations of amentoflavone or PD98059 for $48 \mathrm{~h}$, respectively. Relative NF-kB activity was investigated with NF-kB reporter gene assay and rectified with cell viability, which was obtained by MTT assay as previously described (7).

Western blotting assay. $3 \times 10^{6}$ SK-Hep1 cells were cultured overnight in a $10 \mathrm{~cm}$ dish, and then treated with different concentrations of amentoflavone or $10 \mu \mathrm{M}$ PD98059 for $48 \mathrm{~h}$, respectively. After treatments, total protein from cells was isolated and collected by using lysis buffer (50 mM Tris- $\mathrm{HCl} \mathrm{pH} 8.0,120 \mathrm{mM} \mathrm{NaCl}, 0.5 \% \mathrm{NP}-40$, and $1 \mathrm{mM}$ phenylmethanesulfonyl fluoride) and centrifugation. Protein levels of MMP-9, XIAP, VEGF, Cyclin-D1, ERK, and pERK were evaluated with Western blotting assay as described by Wang et al. (14). Quantification of protein bands was performed by using Bio-Rad Image Lab software (Bio-Rad Laboratories, Inc., CA, USA)

Invasion assay. Transwell insert with $8 \mu \mathrm{m}$ pore size was coated with $50 \mu \mathrm{l}$ matrigel solution ( 1 to 1 matrigel with DMEM) and placed overnight at $37^{\circ} \mathrm{C} .3 \times 10^{6} \mathrm{SK}-\mathrm{Hep} 1$ cells were incubated overnight in a $10 \mathrm{~cm}$ dish and then treated with different concentrations of amentoflavone or $10 \mu \mathrm{M}$ PD98059 for $48 \mathrm{~h}$, respectively. After treatments, $1 \times 10^{6}$ viable cells were harvested with centrifugation and resuspended in $1 \mathrm{ml}$ serum free DMEM. $100 \mu \mathrm{l}$ cell suspension was added into the apical chamber of transwell insert and maintained for $48 \mathrm{~h}$. Effect of treatments on cell invasion was evaluated with cell invasion assay as described by Lai et al. (15). Light Nikon ECLIPSE Ti-U microscope was used for photography of invaded cells at $\times 100$ and number of invaded cells was quantified by using ImageJ software version 1.50 (National Institutes of Health, Bethesda, MD, USA).

Statistical analysis. Analysis of variance (ANOVA) was used to test significance of difference between each experimental group. Data was expressed as mean \pm stand error. $p$-Value less than 0.05 was considered significant.

\section{Results}

Amentoflavone induces cytotoxicity and $N F-k B$ inactivation in SK-Hep1 cells. SK-Hep1 cells after exposed to various concentrations of amentoflavone were analyzed for cell viability. Cell viability was significantly reduced in a dose dependent manner, by $14 \%-58 \%$ compared to control (Figure 1A). Figure $1 \mathrm{~B}$ demonstrates that increasing the amentoflavone 

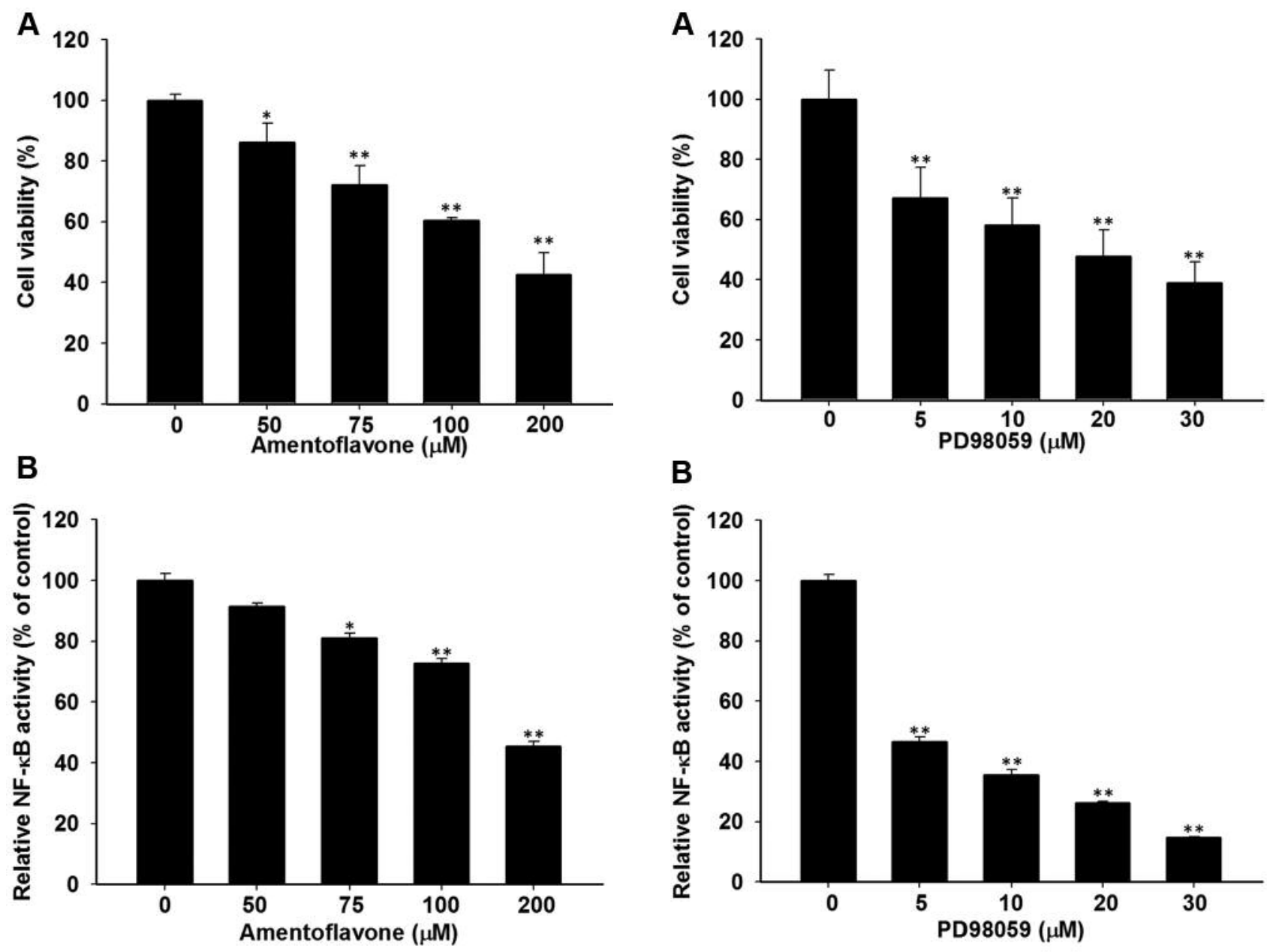

Figure 1. Effect of amentoflavone on cell viability and $N F-k B$ activation in SK-Hepl cells. Cells were treated with different concentrations (0-200 $\mu \mathrm{M}$ in 0.1\% DMSO) of amentoflavone for $48 \mathrm{~h}$. (A) Change of cell viability was evaluated with MTT assay. (B) NF- $k B$ activation was determined with $N F-k B$ reporter gene assay and corrected by using cell viability. $* p<0.05$ and ${ }^{* *} p<0.01$ compared to control (0.1\% DMSO treatment).

concentration significantly inhibited NF-kB activation of SKHep1 cells by $10-55 \%$ as compared to the control.

Blockage of ERK activation reduces cell growth and $N F-k B$ activation in SK-Hepl cells. PD98059, an ERK inhibitor, was used to treat SK-Hep1 cells. Subsequently, change of cell viability and NF-kB activation were investigated with MTT and NF-kB reporter gene assay. Figure 2A shown PD98059 significantly decreases cell growth of SK-Hep1 in a dose-dependent manner, by $33-62 \%$ compared to control. Figure 2B indicates NF-kB activation of SK-Hep1 cells was also significantly reduced by PD98059 treatment compared to control.
Amentoflavone suppresses expression of tumor progressionassociated proteins through inhibition of ERK phosphorylation in SK-Hepl cells. Western blotting assay was used to evaluate protein levels of MMP-9, XIAP, VEGF, Cyclin-D1, and pERK of SK-Hep1 cells after PD98059 or amentoflavone treatment. Figure 3A shows that PD98059 inhibits protein levels of MMP9, XIAP, VEGF and cyclin-D1 by 0.4-0.8-fold as compared to those of control. In addition to the inhibition of tumor progression-associated protein expression, amentoflavone also decreased protein levels of pERK in Figure 3B.

Both amentoflavone and PD98059 inhibit the invasive ability of SK-Hepl cells. Figure 4A indicates that amentoflavone 

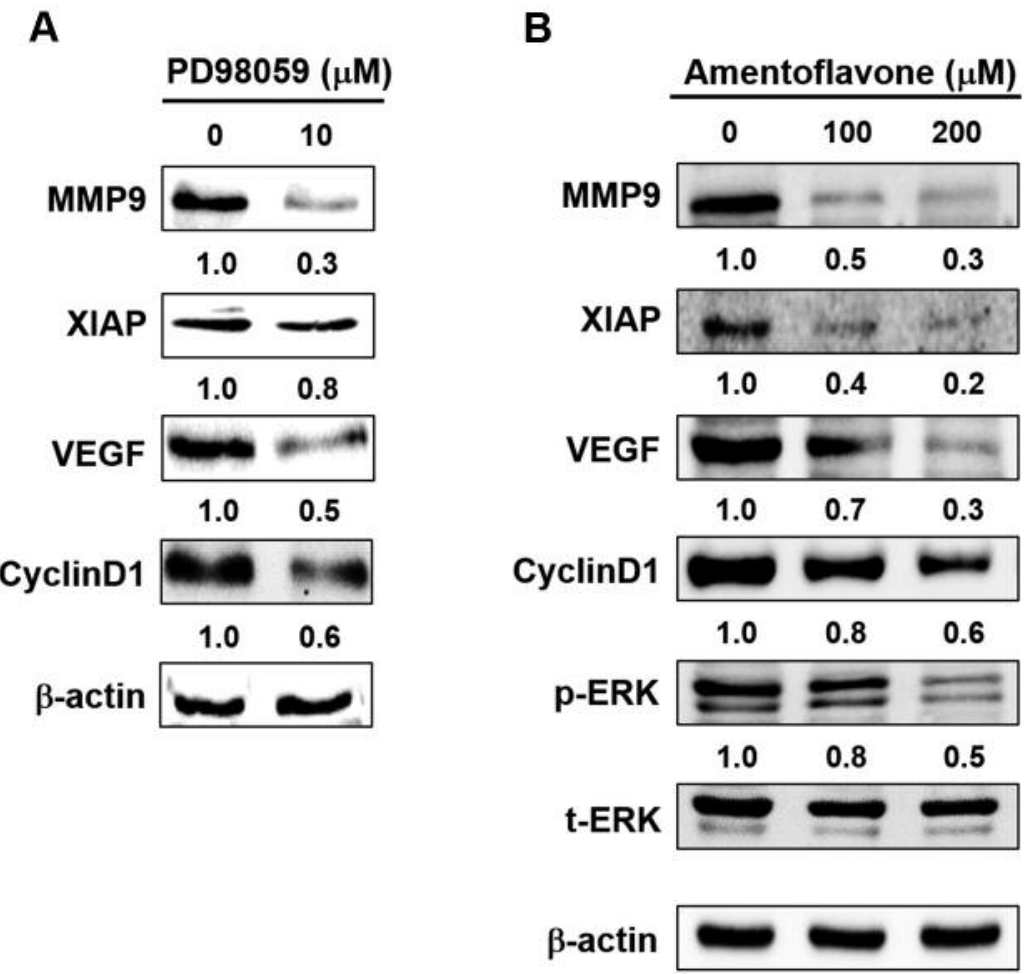

Figure 3. Effect of PD98059 and amentoflavone on the expression of tumor progression-associated proteins in SK-Hep1 cells. Cells were treated with $10 \mu \mathrm{M}$ PD98059, or different concentrations $(0,100,200 \mu \mathrm{M})$ of amentoflavone for $48 \mathrm{~h}$, respectively. Protein levels of MMP-9, XIAP, VEGF, cyclin-D1, and pERK were evaluated with western blotting assay. (A) PD98059 treatment, (B) Amentoflavone treatment.

significantly decreased the number of invaded cells by $40-$ $80 \%$ as compared to control. We also found that $10 \mu \mathrm{M}$ PD98059 significantly reduces cell invasion by $90 \%$ as compared to control (Figure 4B).

\section{Discussion}

In our previous study amentoflavone induced cell cycle arrest and apoptosis leading to inhibition of breast cancer cell growth (16). However, the anti-cancer effect and the mechanism of action of amentoflavone in HCC is unknown. In this study, we demonstrated anti-cancer effect of amentoflavone and the role of ERK inactivation on amentoflavone-induced inhibition of tumor progression in HCC. In previous studies on HCC cell lines, such as SKHep1 and Huh7, we found that inhibition of NF-kB activation impairs tumor progression including antiapoptosis, angiogenesis, proliferation, and metastasis. In addition, blockage of ERK activation may also downregulate NF-kB-modulated tumor progression $(5,7)$.

Sorafenib, the oral multi-kinase inhibitor used for the treatment of $\mathrm{HCC}$, has been shown to diminish HCC growth through inhibition of Raf/MEK/ERK signaling transduction (17).
The effect of sorafenib on NF-kB-modulated tumor progression in $\mathrm{HCC}$ was demonstrated from our previous studies. Sorafenib inhibits NF-kB-modulated tumor progression by triggering ERK dephosphorylation in HCC both in vitro and in vivo $(7,18)$. In this study, we showed that ERK dephosphorylation is a critical factor in amentoflavone-induced inhibition of tumor progression in SK-Hep1 cells. Pan et al. also indicated that amentoflavone inhibits metastatic potential through suppression of ERK/NF-KB activation in osteosarcoma cells (8).

In conclusion, this study demonstrated that amentoflavone has the potential to inhibit HCC progression through suppression of ERK activation.

\section{Acknowledgements}

This study was supported by grants RD2017-016 and CTU106-P-16 from the National Yang-Ming University Hospital, Yilan and CentralTaiwan University of Science and Technology, Taichung, Taiwan, respectively. This study was supported by Taipei Medical University/Taipei Medical University Hospital (Grant no. TMU105AE1-B49). The Authors acknowledge the technical services provided by Clinical Medicine Research Laboratory of National Yang-Ming University Hospital and Translational Laboratory, Department of Medical Research, Taipei Medical University Hospital. 
A
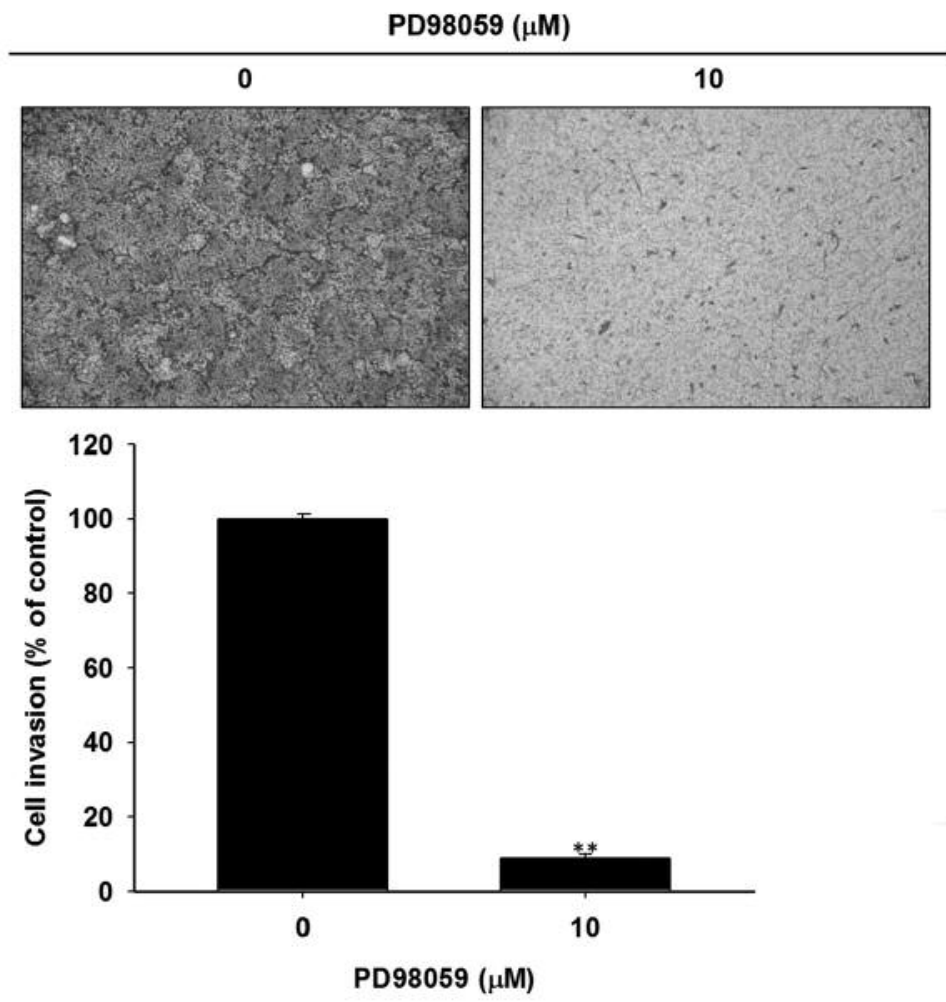

B
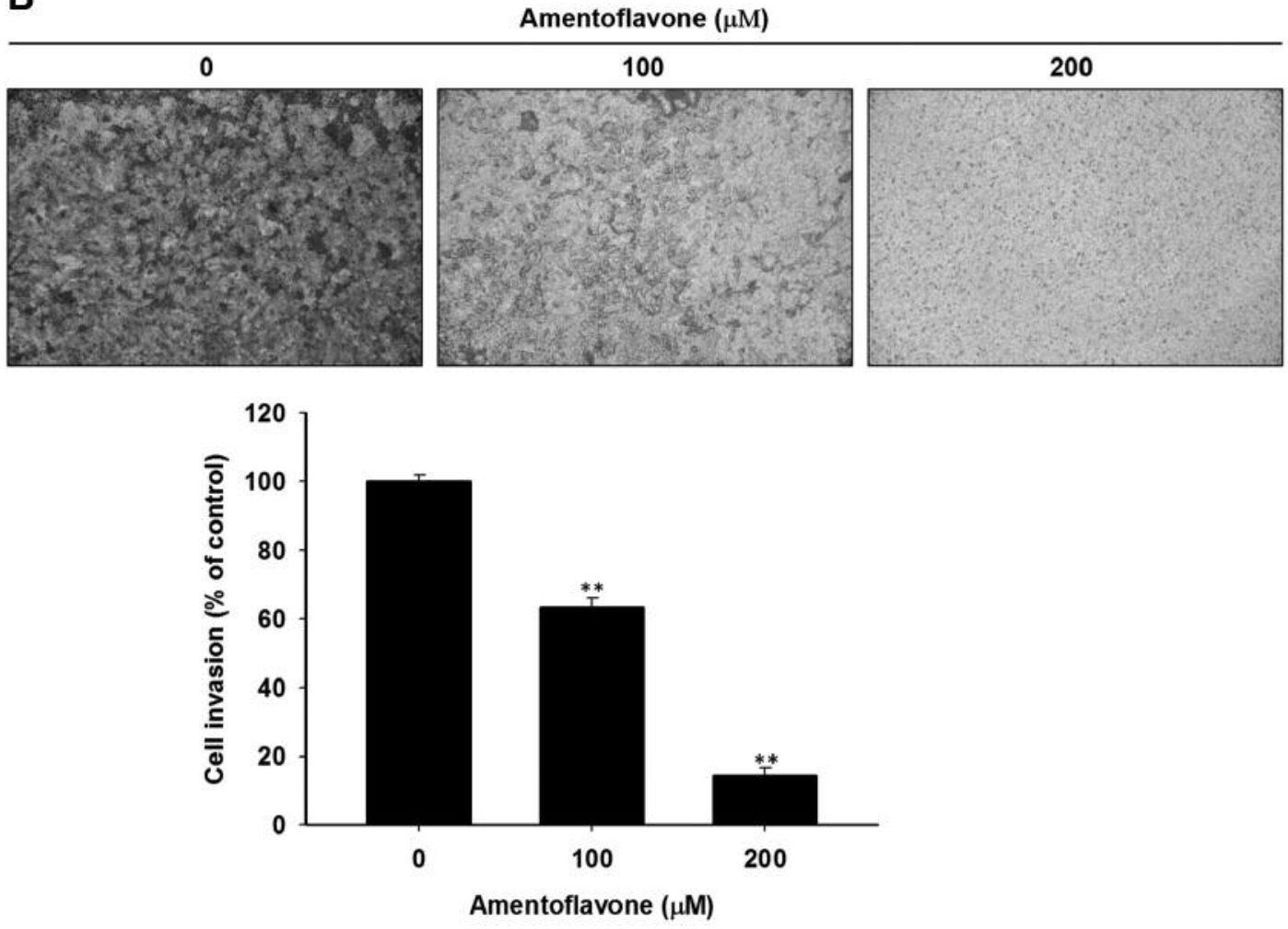

Figure 4. Effect of PD98059 and amentoflavone on cell invasion in SK-Hep1 cells. Cells were treated with $10 \mu M$ PD98059, or different concertation $(0,100,200 \mu M)$ of amentoflavone for 48 h, respectively. Invasive ability of SK-Hep1 cells was determined by using cell invasion assay. (A) PD98059 treatment, (B) Amentoflavone treatment. ${ }^{* *} p<0.01$ as compared to control (0.1\% DMSO treatment). 


\section{References}

1 Roberts PJ and Der CJ: Targeting the Raf-MEK-ERK mitogenactivated protein kinase cascade for the treatment of cancer. Oncogene 26: 3291-3310, 2007.

2 Yang S and Liu G: Targeting the Ras/Raf/MEK/ERK pathway in hepatocellular carcinoma. Oncol Lett 13: 1041-1047, 2017.

3 Schmitz KJ, Wohlschlaeger J, Lang H, Sotiropoulos GC, Malago M, Steveling K, Reis H, Cicinnati VR, Schmid KW and Baba HA: Activation of the ERK and AKT signalling pathway predicts poor prognosis in hepatocellular carcinoma and ERK activation in cancer tissue is associated with hepatitis $\mathrm{C}$ virus infection. J Hepatol 48: 83-90, 2008.

4 Liu YC, Wu RH and Wang WS: Regorafenib diminishes the expression and secretion of angiogenesis and metastasis associated proteins and inhibits cell invasion via NF-kB inactivation in SK-Hep1 cells. Oncol Lett 14: 461-467, 2017.

5 Tsai JJ, Pan PJ and Hsu FT: Regorafenib induces extrinsic and intrinsic apoptosis through inhibition of ERK/NF-kB activation in hepatocellular carcinoma cells. Oncol Rep 37: 1036-1044, 2017.

6 Hoesel B and Schmid JA: The complexity of NF-kB signaling in inflammation and cancer. Mol Cancer 12: 86, 2013.

7 Hsu FT, Liu YC, Chiang IT, Liu RS, Wang HE, Lin WJ and Hwang JJ: Sorafenib increases efficacy of vorinostat against human hepatocellular carcinoma through transduction inhibition of vorinostat-induced ERK/NF-kB signaling. Int J Oncol 45: 177-188, 2014.

8 Pan PJ, Tsai JJ and Liu YC: Amentoflavone inhibits metastatic potential through suppression of ERK/NF- $\mathrm{kB}$ activation in osteosarcoma U2OS cells. Anticancer Res 37: 4911-4918, 2017.

9 Yu S, Yan H, Zhang L, Shan M, Chen P, Ding A and Li SF: A review on the phytochemistry, pharmacology, and pharmacokinetics of amentoflavone, a naturally-occurring biflavonoid. Molecules 22: pii: E299, 2017.

10 Chen WL, Hsieh CL, Chen JH, Huang CS, Chen WT, Kuo YC, Chen CY and Hsu FT: Amentoflavone enhances sorafenibinduced apoptosis through extrinsic and intrinsic pathways in sorafenib-resistant hepatocellular carcinoma SK-Hep1 cells in vitro. Oncol Lett 14: 3229-3234, 2017.
11 Ma CY, Ji WT, Chueh FS, Yang JS, Chen PY, Yu CC and Chung JG: Butein inhibits the migration and invasion of SK-HEP-1 human hepatocarcinoma cells through suppressing the ERK, JNK, p38, and uPA signaling multiple pathways. J Agric Food Chem 59: 9032-9038, 2011.

12 Chen JH, Chen WL and Liu YC: Amentoflavone induces antiangiogenic and anti-metastatic effects through suppression of NF$\mathrm{kB}$ activation in MCF-7 cells. Anticancer Res 35: 6685-6693, 2015.

13 Chiang IT, Liu YC, Hsu FT, Chien YC, Kao CH, Lin WJ, Chung JG and Hwang JJ: Curcumin synergistically enhances the radiosensitivity of human oral squamous cell carcinoma via suppression of radiation-induced NF-kB activity. Oncol Rep 31: 1729-1737, 2014.

14 Wang WH, Chiang IT, Ding K, Chung JG, Lin WJ, Lin SS and Hwang JJ: Curcumin-induced apoptosis in human hepatocellular carcinoma j5 cells: critical role of $\mathrm{ca}(+2)$-dependent pathway. Evid Based Complement Alternat Med 2012: 512907, 2012.

15 Lai KC, Hsu SC, Kuo CL, Ip SW, Yang JS, Hsu YM, Huang HY, $\mathrm{Wu}$ SH and Chung JG: Phenethyl isothiocyanate inhibited tumor migration and invasion via suppressing multiple signal transduction pathways in human colon cancer HT29 cells. J Agric Food Chem 58: 11148-11155, 2010.

16 Pei JS, Liu CC, Hsu YN, Lin LL, Wang SC, Chung JG, Bau DT and Lin SS: Amentoflavone induces cell-cycle arrest and apoptosis in MCF-7 human breast cancer cells via mitochondriadependent pathway. In Vivo 26: 963-970, 2012.

17 Liu L, Cao Y, Chen C, Zhang X, McNabola A, Wilkie D, Wilhelm S, Lynch $\mathrm{M}$ and Carter C: Sorafenib blocks the RAF/MEK/ERK pathway, inhibits tumor angiogenesis, and induces tumor cell apoptosis in hepatocellular carcinoma model PLC/PRF/5. Cancer Res 66: 11851-11858, 2006.

18 Chiang IT, Liu YC, Wang WH, Hsu FT, Chen HW, Lin WJ, Chang WY and Hwang JJ: Sorafenib inhibits TPA-induced MMP-9 and VEGF expression via suppression of ERK/NF- $\mathrm{KB}$ pathway in hepatocellular carcinoma cells. In Vivo 26: 671-681, 2012.

Received February 6, 2018

Revised February 23, 2018

Accepted February 26, 2018 